\title{
The New World of Programmable Photonics
}

\author{
Wim Bogaerts ${ }^{1}$, David A. B. Miller ${ }^{2}$ and Jose Capmany ${ }^{3}$ \\ ${ }^{1}$ Ghent University - imec, Photonic Research Group, Department of Information Technology, Gent, Belgium. \\ Center of Nano and Biophotonics, Ghent University, Belgium. (wim.bogaerts@ugent.be) \\ ${ }^{2}$ Ginzton Laboratory, Spilker Building, Stanford University, Stanford, California 94305-4088, USA. (dabm@ee.stanford.edu) \\ ${ }^{3}$ ITEAM Research Institute, Universitat Politecnica de Valencia, Valencia 46022, Spain (jcapmany@iteam.upv.es)
}

\begin{abstract}
Programmable photonics defines the technologies to manipulate light under software control on a microscopic scale, often on a photonic integrated circuit, offering the optical equivalent of field-programmable-gate-arrays in electronics. Programmable photonics enables uniquely new applications, but can also make the photonic ecosystem more widely accessible.

Index Terms-Photonics, Programmable Circuits
\end{abstract}

\section{INTRODUCTION}

In the past decade photonic integrated circuits (PIC) have found their way into many applications, supported by a number of different technology platforms (silicon, III-V, dielectrics or polymers), and an ever increasing integration density and circuit complexity. Today, most photonic integrated circuits are created for a specific purpose, such as transceivers, switches, spectrometers or sensors. As they can only perform that one function, they could be called application-specific photonic integrated circuits (ASPIC). In that respect they are similar to electronic ASICs, but also to dedicated, application-specific free-space optical systems.

In contrast, in the past few years generic architectures of programmable photonic ICs have emerged, with connectivity and functionality defined during operation, usually with electro-optic tuning elements [1]. The connectivity within a passive waveguide network with tunable $2 \times 2$ couplers and phase shifters can, for example, now be adjusted and controlled in software during operation. With appropriate mesh scales, such programmable waveguide meshes can then perform arbitrary linear operations between input and output ports. When path delays are added, this transformation can be wavelength dependent, allowing programmable wavelength filters. These circuits are the optical equivalent of electronic field-programmable gate arrays (FPGA). Different names have been coined for such circuits, such as linear optical processors, field-programmable linear arrays, programmable photonic circuits, programmable nanophotonic processors, reconfigurable optical circuits, etc.

A conceptual schematic of a generic programmable photonic circuit is shown in Fig.1a. A programmable linear optical circuit connects optical input/outputs to active elements such as photodetectors and modulators. The linear processing core

This work has received funding from the European Research Council under grant agreements No 725555 (PhotonicSWARM) and No 741415 (UMWPCHIP), the European Horizon 2020 programme under grant agreement No 780283 (MORPHIC) and the US Air Force Office of Scientific Research under award number FA9550-17-1-0002. itself is a passive waveguide circuit that can be electrically reconfigured. Forward-propagating topologies based on MachZehnder interferometer networks (Fig. 1b) map a set of input waveguides to output waveguides [3], [4]. More complex architectures, like the meshes of coupled waveguide loops, introduce optical feedback and delay lines for wavelength filters [5], [6]. Modulators and photodetectors act as interfaces for high-speed RF signals, enabling optical processing of microwave signals.

Though some basic concepts were proposed in the 1990s [2], PIC technology only got sufficiently mature in the past few years to fabricate these large circuits, and algorithms and architectures for configuration and control emerged only recently. Since then, programmable photonics has grown rapidly, especially because there are many potential applications that benefit uniquely from these new circuits. The potential of

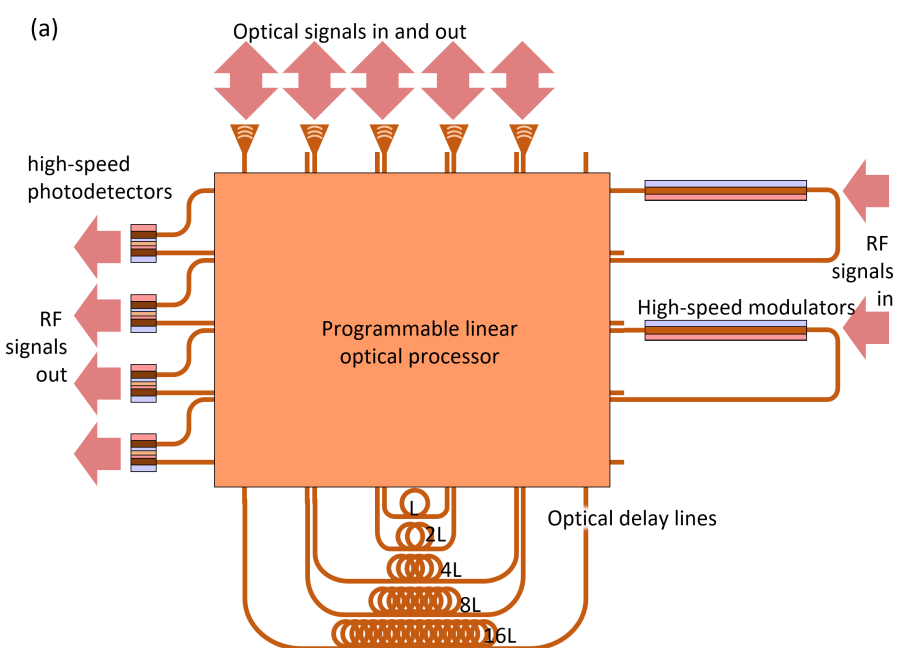

(b)

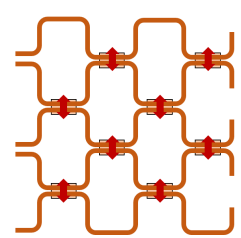

(c)

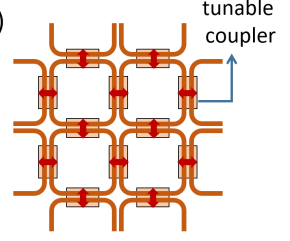

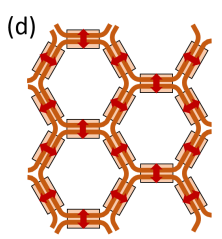

Fig. 1. Conceptual generic programmable photonic circuit. (a) A linear optical processor connects the optical inputs and outputs with active elements such as modulators and photodetectors. The linear circuit can have either (b) a feed-forward architecture of coupled Mach-Zehnder interferometers [2]-[4], or (c-d) a network with rectangular [5] or hexagonal loops. [6] 


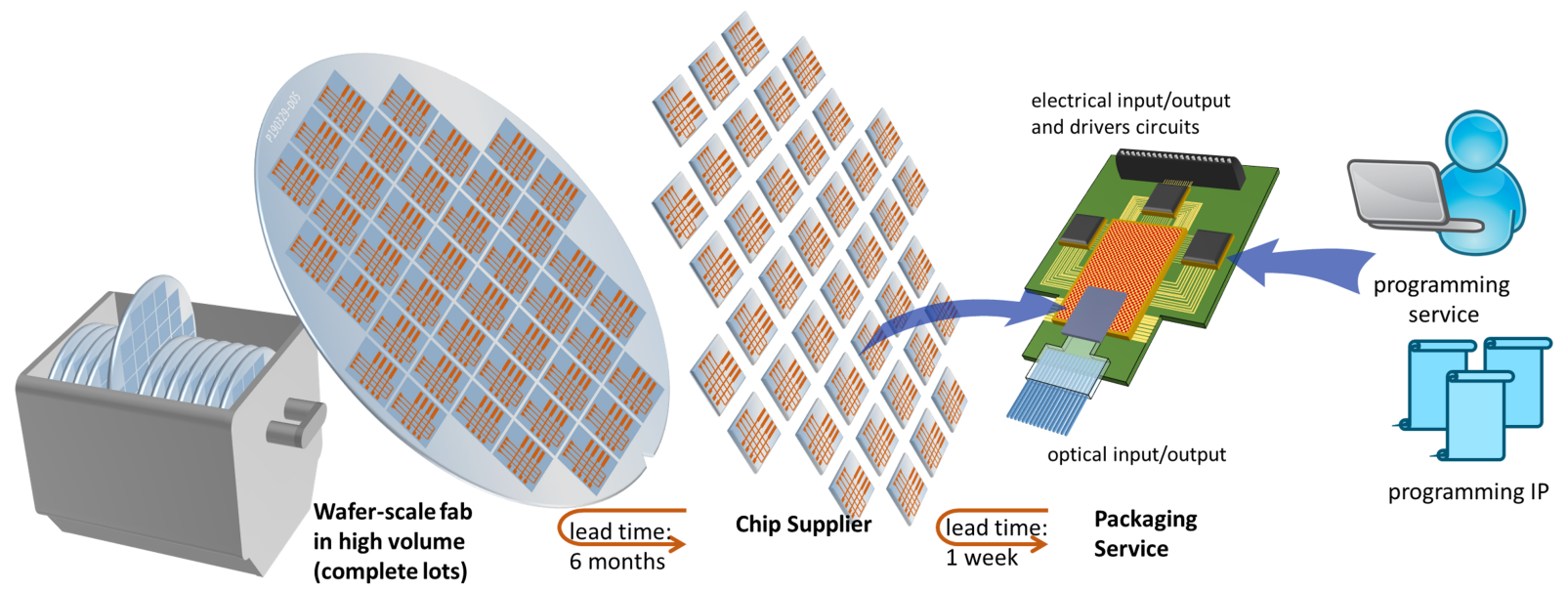

Fig. 2. Generic programmable PICs have a different manufacturing and supply model than ASPICs. They can be fabricated in larger volumes, and supplied off-the-shelf at short notice. Around these chips a new ecosystem of packaging and programming services can evolve.

optics for computation, making use of the inherent nature of photons to perform massively parallel linear processing, maps directly on the needs for quantum information processing, microwave photonics and accelerators for machine learning.

These concepts are not limited to photonic integrated circuits, and can go beyond the plane of the chip, giving powerful architectures for real-time optical transformations between multiple planes. The strong interest in optical beam forming for applications in LiDAR, free-space communication and augmented/virtual reality reaffirms the importance and potential impact of such programmable photonics.

\section{A NEW ECOSYSTEM}

Programmable photonics comes with its own set of challenges. The circuits may be larger and more complex than equivalent ASPICs. The correspondingly large numbers (100s1000s) of phase shifters need accurate control, imposing aggressive requirements on electronics, wiring, packaging and system integration. The control and supervision algorithms are far from trivial, ranging from purely local feedback loops to pre-calibrated global configuration. In the longer term, the development of suitable specification and programming software carrying out similar tasks to that of VDHL in electronics will be critical. This creates opportunities for innovation and generation of new layers of intellectual property in integrated photonics.

Just as FPGAs have transformed the ecosystem of electronic ICs, programmable photonic chips can have a similar impact on the PIC supply chain. ASPICs today struggle with long development times and high production costs, as the aggregate production volume for photonic chips is still very low compared to electronics. Development of new applications making use of integrated photonics is therefore prohibitively expensive. As illustrated in Fig. 2, generic programmable PICs can break this chicken-and-egg problem. As the functionality in these chips can be programmed, implementing functionality is decoupled from the long chip fabrication cycle, and it becomes possible to prototype new applications in a matter of weeks, rather than months, using off-the-shelf programmable PICs. And because these PICS can be used for many applications, they can be manufactured in larger volumes, bringing down the cost. As with FPGAs and ASICS, these programmable PICs may never reach the ultimate performance as dedicated ASPICs, but they might still provide sufficient functionality at an acceptable cost for many low-volume applications. Furthermore, their real-time programmability may open new opportunities for adaptive, self-configuring and self-stabilizing systems. And like FPGAs, a programmable PIC platform makes photonics accessible to a broader audience, which might stimulate a wave of innovation from the maker community.

\section{CONCLUSION}

Programmable photonics are opening up an entire new space in optics, enabling manipulation of light in software for a multitude of applications, and new systems that configure themselves to the problems. Though programmable PICs introduce significant scaling challenges for chip fabrication processes, packaging, interfaces and control, they could significantly lower the adoption threshold for integrated photonics and open many exciting new applications.

\section{REFERENCES}

[1] D. Pérez, I. Gasulla, and J. Capmany, "Programmable multifunctional integrated nanophotonics - Review Article," De Gruyter - Nanophotonics 2018, vol. 7, no. 8, pp. 1351-1371, 2018.

[2] M. Reck, A. Zeilinger, H. J. Bernstein, and P. Bertani, "Experimental realization of any discrete unitary operator," Phys. Rev. Lett., vol. 73, no. 1 , pp. 58-61, 71994.

[3] D. A. B. Miller, "Self-configuring universal linear optical component," Photonics Research, no. 1, pp. 1-15, 2013.

[4] A. Ribeiro, A. Ruocco, L. Vanacker, and W. Bogaerts, "Demonstration of a 4 X 4-port universal linear circuit," Optica, vol. 3, no. 12, p. 1348, 2016.

[5] L. Zhuang, C. G. H. Roeloffzen, M. Hoekman, K. Boller, and A. J. Lowery, "Programmable photonic signal processor chip for radiofrequency applications," vol. 2, no. 10, pp. 1-6, 2015.

[6] D. Pérez, I. Gasulla, L. Crudgington, D. J. Thomson, A. Z. Khokhar, K. Li, W. Cao, G. Z. Mashanovich, and J. Capmany, "Multipurpose silicon photonics signal processor core," Nature Communications, vol. 8, no. 1, pp. 1-9, 2017. 